\title{
Preoperative albumin-to-fibrinogen ratio predicts severe postoperative complications in elderly gastric cancer subjects after radical laparoscopic gastrectomy
}

Xuexue You', Qun Zhou', Jie Song ${ }^{1}$, Linguang Gan², Junping Chen ${ }^{2}$ and Huachun Shen ${ }^{2 *}$

\begin{abstract}
Background: A high prevalence of postoperative complications is closely associated with a worse short- and longterm outcome. This current study aimed to investigate potential risk factors including albumin-to-fibrinogen ratio (AFR) for severe postoperative complications (SPCs) in surgical gastric cancer (GC) patients.

Methods: Elderly patients ( $\geq 65$ years) with primary GC who underwent elective radical laparoscopic gastrectomy under general anesthesia were included. According to the Clavien-Dindo classification system, the severity of complications was assessed from Grade I to V and SPCs were defined as C-D Grade $\geq$ Illa. The clinicopathological features, operative-associated characteristics, postoperative recovery and laboratory tests were compared between patients with or without SPCs. Receiver operating characteristic (ROC) curve analysis using Youden's Index was established for determining the predictive value and cut-off threshold of AFR for SPCs. Binary univariate and multivariate logistic regression models were used to assess factors influencing SPCS.
\end{abstract}

Results: A total of 365 elderly GC patients were finally included in the analysis, of which $52(52 / 365,14.2 \%)$ patients had developed SPCS within postoperative 30 days. Preoperative AFR level predicted SPCs in surgical GC patients with an AUC of 0.841 , a sensitivity of $76.36 \%$ and a specificity of $80.77 \%$, respectively $(P<0.001)$. The multivariate analysis revealed that a lower AFR level (OR: 1.94, 95\% Cl: 1.09-3.36, $P=0.017)$ and an older age (OR: 1.81, 95\% Cl: 1.06-3.04, $P=0.023$ ) were two independent predictive factors for SPCs in surgical GC patients.

Conclusions: Preoperative AFR level is a useful predictor for SPCs in elderly GC subjects after radical laparoscopic gastrectomy.

Keywords: Gastric cancer, Severe postoperative complications, Predictor, Albumin, Fibrinogen

\section{Background}

Gastric cancer (GC) is the fourth most common malignant neoplasm with an increasing incidence and it ranks second in cancer mortality worldwide [1]. Due to the high prevalence, recurrence rate and mortality rate, GC has become a significant global health problem [2]. As for resectable GC,

\footnotetext{
* Correspondence: shenhuachun_nb@sina.com

${ }^{2}$ Department of Anesthesiology, HwaMei Hospital, University of Chinese Academy of Sciences, No.41 Xibei Road, Haishu District, Ningbo, Zhejiang Province, China

Full list of author information is available at the end of the article
}

surgical resection with systematic lymphadenectomy remains the standard treatment [3]. Advances in surgical techniques, instruments, and experiences have led to a corresponding decrease of postoperative complications, as well as improved outcomes [4]. However, a high prevalence of postoperative complications, with ranges from 14.3 to $34.0 \%$, is closely associated with an increased economic burden, a prolonged hospital stay, a worse short- and longterm outcome [5]. Therefore, to improve the overall prognoses of GC patients, robust biomarkers for predicting severe postoperative complications (SPCs) after radical

(c) The Author(s). 2019 Open Access This article is distributed under the terms of the Creative Commons Attribution 4.0 International License (http://creativecommons.org/licenses/by/4.0/), which permits unrestricted use, distribution, and 
gastrectomy could help with the risk identification, follow-up facilitation and more aggressive postoperative care. Despite the significant improved perioperative managements, multidisciplinary therapeutic strategies and surgical techniques, a high prevalence of SPCs still remains to some extent [6].

Albumin (Alb), as a negative acute phase protein and a nutritional biomarker, usually decreases after surgery due to the surgery stress and increased capillary leakage [7]. Preoperative serum Alb is reported to be a predictive factor for postoperative recovery [8] and long-term survival [9] in GC patients. Another study has revealed that postoperative decrease of serum Alb expression can serve as a predictor for short-term complications in GC patients [10]. Preoperative low serum Alb expression was reported to be a potential risk factor for SPCs in elderly GC subjects [11]. Fibrinogen (Fib) is an essential protein for coagulation cascade as well as an acute-phase reaction protein in response to systemic inflammation [12]. Kanda et al. have indicated that Fib level is associated with tumor stage, metastasis, and outcomes in solid tumors, including GC [13]. Moreover, low preoperative Fib level is suggested as a potential risk factor for neurological complications after cardiac surgeries [14]. However, whether Alb or Fib can serve as a predictor for SPCs in GC patients still remains controversial. Alb-to-Fib ratio (AFR), a combination of Alb and Fib, has been reported to be a prognostic factor for non smallcell lung cancer patients $[15,16]$. This study focused on the potential prognostic role of AFR for SPCs in GC patients.

\section{Methods}

\section{Patients}

This retrospective study was approved by the Medical Institutional Ethics Committee of Jiangxi province and our hospital. Elderly patients with primary GC who underwent elective radical laparoscopic gastrectomy under general anesthesia at the Department of anesthesiology, Jiangxi maternal and child health hospital from March 2014 to March 2018 were included. All the participants provided written informed consent. Inclusion criteria were described as follows: 1 ) elderly patients aged between 65 and 80 years; 2) diagnosed with primary GC which was supported by operative and pathological results; 3) undergoing systemic evaluation before the surgery including computed tomography (CT) image and 4) undergoing elective radical laparoscopic gastrectomy for the first time. The exclusion criteria were described as follows: 1) with tumor distant metastasis; 2) undergoing emergency operations due to complications (bowel obstruction, perforation, etc.) before the surgery; 3) with preoperative neoadjuvant treatment (radiotherapy or chemotherapy); 4) combined with other malignancies; 5) undergoing laparotomy or laparoscopic conversion to laparotomy; 6) with incomplete data. A total of 402 patients were initially enrolled and 37 patients were excluded according to the exclusion criteria (4 with tumor distant metastasis, 5 underwent emergency operations, 3 combined with other malignancies, 10 underwent laparotomy or laparoscopic conversion to laparotomy, 15 with data missed).

\section{Study design}

The operative procedures for GC (the extent of gastrectomy and lymph node dissection) in this study was according to the Japanese Gastric Cancer Treatment Guidelines 2010 (ver. 3) [17]. All the enrolled patients were operated by the same experienced surgeons with the same perioperative managements. The pathological diagnosis was performed following the guidelines of the American Joint Committee on Cancer (AJCC) TNM Staging System for GC [18].

\section{Data collection}

The following data were extracted and recorded from our database: 1) clinicopathological features, including age, gender, body mass index (BMI), American Society of Anesthesiologists (ASA) grade, comorbidities, smoking and drinking habits, abdominal surgery history, tumor location, histologic type, pathological type, tumor size and TNM stage; 2) operative-associated characteristics including extent of resection, operation time, estimated blood loss, intraoperative fluid utilization and perioperative blood transfusion; 3) postoperative recovery including time to first flatus, ambulation, first liquid intake and first soft intake; 4) laboratory tests.

Assessment and definition of postoperative complications The primary end point of this study was set as the occurrence of postoperative complications within postoperative 30 days [19]. According to the Clavien-Dindo classification system, the severity of complications was assessed from Grade I to V and SPCs were defined as C-D Grade $\geq$ IIIa [20]. If the patient had multiple complications, the grading was performed based on the most serious complication. Each patient was assessed for C-D grading by two independent experienced surgeons and divergences were solved by discussion. Enrolled patients were subdivided into SPCs group and non-SPCs group according to the presence of SPCs within postoperative 30 days.

\section{Laboratory tests}

Fasting blood samples from each participant were obtained on 1 day before the operation. Blood cell analyses including hemoglobin ( $\mathrm{Hb})$, white blood cell (WBC), platelet (Plt) and hematocrit (Hct), biochemistry analyses including creatinine and urea were determined in the laboratory of our hospital. The serum expressions of inflammatory cytokines including tumor necrosis factor- $\alpha$ (TNF- $\alpha$ ), C-reactive protein (CRP) and interleukin-6 (IL- 
6) were measured using the method of enzyme-linked immunesorbent assays (ELISA). The measurement procedures were performed according to the manufacturers' instructions (R\&D Systems, Minneapolis, MN, USA). AFR was calculated by Alb (g/L)/Fib (g/L) ratio.

\section{Statistical analysis}

Statistical analysis was performed using SPSS 22.0 (SPSS Inc., IA, USA) and GraphPad Prism 5.0 (GraphPad Inc., CA, USA). All variables are expressed as means \pm standard deviation (SD) or numbers with percentage (n, \%). Continuous variables were compared using MannWhitney $\mathrm{U}$ test or Student $\mathrm{t}$ test, whereas categorical variables using Chi-square test or Fisher exact test as appropriate. Receiver operating characteristic (ROC) curve analysis using Youden's Index was established for determining the predictive value and cut-off threshold of AFR for SPCs. Binary univariate and multivariate logistic regression models were used to assess factors influencing SPCs. Following univariate analysis, potential risk factors $(P<0.1)$ were selected for multivariate analysis using the multivariate logistic regression model with binary stepwise regression method. A two-sided $P$ value of $<0.05$ was considered statistically significant.

\section{Results}

\section{Patient characteristics}

According to the inclusion and exclusion criteria, 365 patients were enrolled in the analysis. The mean age of this study population was 73.1 years and the majority $(275 / 365,75.3 \%)$ were male patients. Among these 365 available participants, 52 were categorized into SPCs group with a prevalence of $14.2 \%(52 / 365)$ and the remaining 313 were categorized into non-SPCs group. The actual number and frequency of each complication in SPCs group are shown in Table 1. Of these postoperative events, pulmonary complications $(n=11,3.0 \%)$, postoperative bleeding $(n=9,2.5 \%)$, intra-abdominal infection $(n=8,2.2 \%)$, bowel obstruction $(n=6,1.6 \%)$, wound infection $(n=5,1.4 \%)$ and anastomotic leakage $(n=4,1.1 \%)$ are the most frequent. The demographic and clinical characteristics of enrolled GC patients associated with SPCs are shown in Table 2. As a result, those patients with an older age and a higher ASA grade were more likely to suffer SPCs $(P<0.05)$. The presence of preoperative comorbidities (hypertension and diabetes) was closely associated with an increased risk of SPCs $(P=$ 0.013 and 0.019 , respectively). The SPCs group experienced significantly longer operation time $(P=0.023)$, higher estimated blood loss $(P=0.012)$ and intraoperative fluid utilization $(P=0.007)$. No statistical differences were observed between SPCs and non-SPCs groups in gender, BMI, hyperlipidaemia, smoking and drinking habits, abdominal surgery history, tumor location, histologic type,
Table 1 Number and frequency of SPCS

\begin{tabular}{ll}
\hline Complications & $\mathrm{n}(\%)$ \\
\hline Total & 52 \\
Pulmonary complications & 11 \\
Postoperative bleeding & 9 \\
Intra-abdominal infection & 8 \\
Bowel obstruction & 6 \\
Wound infection & 5 \\
Anastomotic leakage & 4 \\
Thrombosis & 2 \\
Heart failure & 2 \\
Others & 5
\end{tabular}

SPCS Severe postoperative complications

pathological type, tumor size, TNM stage, extent of resection, perioperative blood transfusion, time to first flatus, ambulation, first liquid intake and first soft intake $(P>0.05)$.

\section{Laboratory tests and SPCs}

Table 3 shows the results of preoperative laboratory tests in surgical patients with or without SPCs. Patients who suffered SPCs had higher preoperative expressions of CRP $(P=0.012)$ and TNF- $\alpha(P=0.016)$ than those who did not. Moreover, those patients with a lower preoperative AFR level were more likely to develop SPCs $(P<$ $0.001)$. There were no significant differences in blood cell analyses, IL-6, Alb, Fib, creatinine and urea between the two groups $(P>0.05)$.

\section{AFR and SPCs}

ROC curve analysis was performed to evaluate the predictive value of AFR for SPCs in GC patients. As illustrated in Fig. 1, preoperative AFR level predicted SPCs in surgical GC patients with an AUC of 0.841 , a sensitivity of $76.36 \%$ and a specificity of $80.77 \%$, respectively $(P<0.001)$. Furthermore, an AFR value of 8.49 was set as the optimal cut-off threshold for SPCs based on the Youden's Index. Enrolled patients were then subdivided by AFR based on the cut-off value, high-AFR group (AFR $>8.49$ ) and lowAFR group $(\mathrm{AFR} \leq 8.49)$.

\section{Risk factors for SPCs}

All potential risk factors $(P<0.05$ in Tables 2 and 3, $n=$ 10 in this study, see Table 4) were enrolled in the univariate and multivariate logistic regression analyses. In the univariate analysis, five risk factors with $P$ values $<0.1$ (age, diabetes, operation time, CRP and AFR) were selected for multivariate analysis. The multivariate analysis revealed that a lower AFR level (OR: 1.94, 95\% CI: 1.09-3.36, $P=0.017$ ) and an older age (OR: 1.81, 95\% 
Table 2 Demographic and clinical characteristics of GC patients with SPCs or not

\begin{tabular}{|c|c|c|c|}
\hline \multirow[t]{2}{*}{ Parameters } & \multicolumn{2}{|l|}{ SPCS } & \multirow[t]{2}{*}{$P$-value } \\
\hline & No $(n=313)$ & Yes $(n=52)$ & \\
\hline Age (year) & $72.3 \pm 5.7$ & $77.6 \pm 6.3$ & $<0.001^{*}$ \\
\hline Gender, n (\%) & & & 0.449 \\
\hline Male & 238 & 37 & - \\
\hline Female & 75 & 15 & - \\
\hline $\mathrm{BMI}\left(\mathrm{kg} / \mathrm{m}^{2}\right)$ & $21.2 \pm 2.1$ & $20.9 \pm 1.9$ & 0.331 \\
\hline Comorbidities, n (\%) & & & - \\
\hline Hypertension & 38 & 13 & $0.013^{*}$ \\
\hline Diabetes & 27 & 10 & $0.019^{*}$ \\
\hline Hyperlipidaemia & 22 & 7 & 0.122 \\
\hline Heavy drinkers, n (\%) & 26 & 7 & 0.230 \\
\hline Current smokers, n (\%) & 33 & 10 & 0.072 \\
\hline ASA grade, n (\%) & & & $0.015^{*}$ \\
\hline$|/| \mid$ & 247 & 33 & - \\
\hline III/IV & 66 & 19 & - \\
\hline Abdominal surgery history, n (\%) & 26 & 7 & \\
\hline Tumor location, n (\%) & & & 0.449 \\
\hline Cardia & 32 & 6 & - \\
\hline Pylorus & 201 & 28 & - \\
\hline Corpus & 67 & 14 & - \\
\hline Total & 13 & 4 & - \\
\hline Histologic type & & & 0.177 \\
\hline Differentiated & 221 & 30 & - \\
\hline Undifferentiated & 33 & 8 & - \\
\hline Signet-ring cell carcinoma & 59 & 14 & - \\
\hline Pathological type & & & 0.210 \\
\hline Ulcerative & 273 & 42 & - \\
\hline Non-ulcerative & 40 & 10 & - \\
\hline Tumor size $(\mathrm{cm})$ & $4.3 \pm 1.9$ & $4.5 \pm 2.1$ & 0.489 \\
\hline Extent of resection & & & 0.741 \\
\hline Distal gastrectomy & 185 & 32 & - \\
\hline Total gastrectomy & 128 & 20 & - \\
\hline T stage & & & 0.876 \\
\hline $\mathrm{Tis} / \mathrm{T} 1 / \mathrm{T} 2$ & 130 & 21 & - \\
\hline $\mathrm{T} 3 / \mathrm{T} 4$ & 183 & 31 & - \\
\hline N stage & & & 0.952 \\
\hline NO & 101 & 17 & - \\
\hline $\mathrm{N} 1 / \mathrm{N} 2 / \mathrm{N} 3$ & 212 & 35 & - \\
\hline TNM stage & & & 0.654 \\
\hline I & 77 & 11 & - \\
\hline$\|$ & 89 & 13 & - \\
\hline III & 147 & 28 & - \\
\hline Operation time (min) & $234.6 \pm 31.3$ & $245.7 \pm 38.2$ & $0.023^{*}$ \\
\hline Estimated blood loss (mL) & $187.9 \pm 78.8$ & $217.2 \pm 70.6$ & $0.012^{*}$ \\
\hline
\end{tabular}


Table 2 Demographic and clinical characteristics of GC patients with SPCs or not (Continued)

\begin{tabular}{llll}
\hline Parameters & \multicolumn{1}{l}{ SPCs } & Yes $(n=52)$ & -value \\
\cline { 2 - 3 } & No $(n=313)$ & $2014.3 \pm 306.4$ & $0.007^{*}$ \\
\hline Intraoperative fluid utilization $(\mathrm{mL})$ & $1910.3 \pm 245.3$ & 16 & 0.694 \\
Perioperative blood transfusion, $\mathrm{n}(\%)$ & 105 & $3.0 \pm 0.9$ & 0.362 \\
Time to first flatus (d) & $2.9 \pm 0.7$ & $2.2 \pm 0.6$ & 0.332 \\
Time to ambulation (d) & $2.1 \pm 0.7$ & $4.0 \pm 1.3$ & 0.555 \\
Time to first liquid intake (d) & $3.9 \pm 1.1$ & $5.3 \pm 1.5$ & 0.616 \\
Time to first soft intake (d) & $5.4 \pm 1.3$ & &
\end{tabular}

$P$-values were calculated by Chi-square test, Fisher exact test, Mann-Whitney $\mathrm{U}$ or $\mathrm{t}$ test

GC Gastric cancer, SPCS Severe postoperative complications, BMI Body mass index, ASA American Society of Anesthesiologists

${ }^{*} P$ value $<0.05$

CI: $1.06-3.04, P=0.023)$ were two independent predictive factors for SPCs in surgical GC patients.

\section{Discussion}

In this present study, we observed that preoperative AFR level and age were two independent predictive factors for SPCs in GC patients undergoing elective radical laparoscopic gastrectomy. Our study reported a prevalence of SPCs of $14.2 \%$ in surgical GC patients, which was a little higher than $10.2 \%$ by Zhang et al. [21] and $8.1 \%$ by Kang et al. [11]. Another study by Fukuda et al. has reported a prevalence of $13.2 \%$ [22], which is quite in accordance with our results. In our consideration, the different sample sizes, age ranges, inclusion and exclusion criteria, SPCs evaluation deviations and some missed data might be possible explanations for the different results.

Table 3 Laboratory tests and SPCs in GC patients

\begin{tabular}{llll}
\hline $\begin{array}{llll}\text { Preoperative } \\
\text { laboratory tests }\end{array}$ & \multicolumn{2}{l}{ SPCs } & P-value \\
\cline { 2 - 3 } & No $(n=313)$ & Yes $(n=52)$ & \\
\hline Hb $(\mathrm{g} / \mathrm{L})$ & $117.5 \pm 7.5$ & $116.4 \pm 8.4$ & 0.337 \\
Plt $\left(\times 10^{9} / \mathrm{L}\right)$ & $207.6 \pm 41.2$ & $214.3 \pm 49.7$ & 0.293 \\
WBC $\left(\times 10^{9} / \mathrm{L}\right)$ & $7.1 \pm 2.2$ & $6.9 \pm 1.9$ & 0.537 \\
$\mathrm{Hct}$ & $0.43 \pm 0.07$ & $0.42 \pm 0.06$ & 0.332 \\
$\mathrm{CRP}(\mathrm{mg} / \mathrm{L})$ & $6.3 \pm 3.1$ & $7.5 \pm 3.5$ & $0.012^{*}$ \\
$\mathrm{IL}-6(\mathrm{pg} / \mathrm{mL})$ & $15.7 \pm 7.1$ & $16.3 \pm 6.6$ & 0.569 \\
TNF-a $(\mathrm{nmol} / \mathrm{L})$ & $7.6 \pm 1.9$ & $8.3 \pm 2.1$ & $0.016^{*}$ \\
Creatinine $(\mathrm{mmol} / \mathrm{L})$ & $83.1 \pm 17.3$ & $82.6 \pm 18.4$ & 0.848 \\
Urea $(\mathrm{mmol} / \mathrm{L})$ & $6.4 \pm 1.8$ & $6.2 \pm 1.7$ & 0.455 \\
Albumin $(\mathrm{g} / \mathrm{L})$ & $39.2 \pm 5.5$ & $37.9 \pm 6.2$ & 0.122 \\
Fibrinogen $(\mathrm{mg} / \mathrm{dL})$ & $3.6 \pm 1.3$ & $3.9 \pm 1.5$ & 0.134 \\
AFR & $9.9 \pm 2.2$ & $7.4 \pm 2.1$ & $<0.001^{*}$ \\
\hline P-valus were & &
\end{tabular}

$P$-values were calculated by Mann-Whitney $U$ or $t$ test

GC Gastric cancer, SPCS Severe postoperative complications, Hb Hemoglobin, Plt Platelet, WBC White blood cell, Hct Hematocrit, CRP C-reactive protein, IL-6 Interleukin-6, TNF- $a$ Tumor necrosis factor- $a, A F R$ Albumin-to-fibrinogen ratio ${ }^{*} P$ value $<0.05$
It is well known that diabetes closely correlates with postoperative complications. Patients who had comorbidities of diabetes before surgery were associated with a high risk of major postoperative complications after reconstructive microsurgery for head and neck cancer [23]. Furthermore, Saji et al. indicated a comprehensive risk scoring system, which included diabetes mellitus as a component, capable of predicting SPCs in patients with medically operable lung cancer [24]. A previous study by Sung et al. reported that a long operation time $(>3 \mathrm{~h})$ was an independent risk factor for severe and overall postoperative complications, as well as poor surgical outcomes [11]. As reported by recent studies, CRP is suggested to be a valid predictor of postoperative complications after various operations, such as minimally invasive colorectal resection [25], minimally invasive esophagectomy [26] and major noncardiac surgery [27]. A randomized controlled trial has also proved the significantly predictive value of CRP for surgical site infection [28]. Our univariate analyses showed that five variables (age, diabetes, operation time, CRP and AFR) might be potential risk factors for SPCs. However, the results from our multivariate analyses only supported age and AFR as two independent risk factors for SPCs. The different sample sizes, operation types, perioperative managements may explain the different conclusions.

Accumulating evidence has demonstrated that aging is an independent risk factor for postoperative complications following various operation types, including pancreatic resection [29], laparoscopic gastrectomy [30], and roboticassisted pulmonary lobectomies [31]. As expected, our results also supported an older age as an independent risk factor for SPCs. Several studies have indicated that older age is associated with high postoperative morbidity and mortality rates due to increased preoperative comorbidities [32]. We consider that the age-associated gradual loss of reserve capacity (e.g. circulatory, immune system changes) [33] and more preexisting diseases [34] may be possible mechanisms for its predictive value for SPCs in this study. However, there is still no consensus with 


\section{ROC curve analysis of AFR for SPCs}

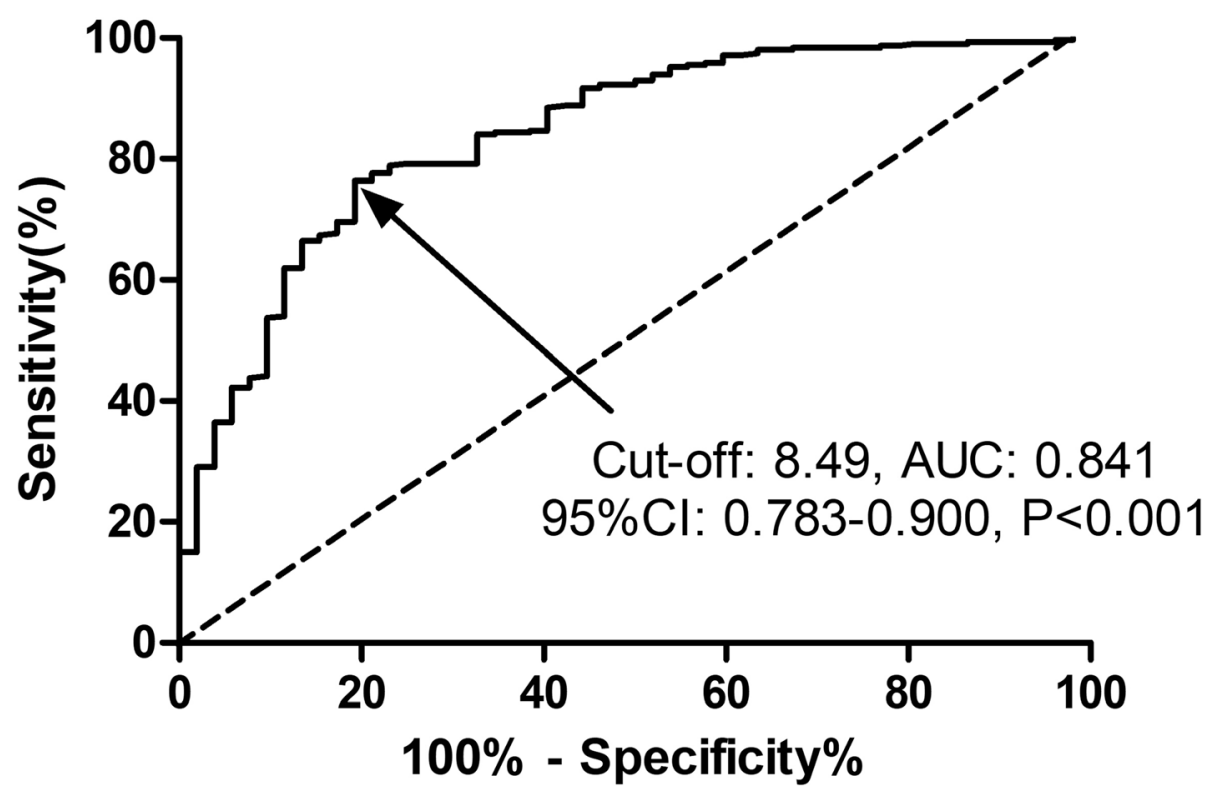

Fig. 1 Predictive value of AFR for SPCs in GC patients by ROC curve analysis. The results indicated preoperative AFR as a potential predictive factor for SPCs in GC patients with an AUC of $0.841,95 \% \mathrm{Cl}$ of $0.783-0.900$, a cut-off value of 8.49 , a sensitivity of $76.36 \%$ and a specificity of $80.77 \%$, respectively $(P<0.001)$. AFR, albumin-to-fibrinogen ratio; SPCs, severe postoperative complications; GC, gastric cancer; ROC, receiver operating characteristic; $\mathrm{AUC}$, the area under the curve; $\mathrm{Cl}$, confidence interval

respect to the cut-offs of ages among the published studies [29].

Alb is a sensitive biomarker for nutritional status evaluation and an acute-phase protein in response to systemic inflammation [35]. Alb expressions are recommended to be a reliable prognostic factor in patients with cancers [36]. Fib, which is synthesized by liver, is a crucial component of blood coagulation system via promoting platelet aggregation. Moreover, Fib is reported to be an important biomarker reflecting systemic inflammation [37] and it serves as a candidate prognostic biomarker in patients with non-small cell lung cancer (NSCLC) [38]. AFR, a ratio of Alb-to-Fib, combines these two biomarkers and amplifies the sensitivity for evaluating inflammation and nutrition status. The combination of Alb and Fib is superior to the single Alb and Fib and it has been widely

Table 4 Risk factors for SPCs by univariate and multivariate logistic regression analyses

\begin{tabular}{|c|c|c|c|c|}
\hline \multirow[t]{2}{*}{ Variables } & \multicolumn{2}{|l|}{ Univariate } & \multicolumn{2}{|l|}{ Multivariate } \\
\hline & OR $(95 \% \mathrm{Cl})$ & $P$ value & OR $(95 \% \mathrm{Cl})$ & $P$ value \\
\hline Age ( $\geq 74$ vs < 74) & $2.22(1.37-3.58)$ & $0.005^{*}$ & $1.81(1.06-3.04)$ & $0.023^{*}$ \\
\hline Hypertension (yes vs no) & $1.41(0.83-2.39)$ & 0.21 & & \\
\hline Diabetes (yes vs no) & $1.72(1.04-2.88)$ & $0.031^{*}$ & $1.29(0.78-2.19)$ & 0.41 \\
\hline ASA grade (I/II vs III/IV) & $0.96(0.59-1.51)$ & 0.81 & & \\
\hline Operation time (high vs low) & $1.71(1.03-2.85)$ & $0.029^{*}$ & $1.54(0.85-2.63)$ & 0.14 \\
\hline Estimated blood loss (high vs low) & $0.94(0.51-1.79)$ & 0.85 & & \\
\hline Intraoperative fluid utilization (high vs low) & $0.92(0.53-1.58)$ & 0.79 & & \\
\hline Preoperative CRP (high vs low) & $1.83(1.11-3.08)$ & $0.024^{*}$ & $1.37(0.79-2.33)$ & 0.25 \\
\hline Preoperative TNF-a (high vs low) & $1.15(0.73-1.77)$ & 0.42 & & \\
\hline $\operatorname{AFR}(\leq 8.49$ vs $>8.49)$ & $2.54(1.52-4.22)$ & $0.001^{*}$ & $1.94(1.09-3.36)$ & $0.017^{*}$ \\
\hline
\end{tabular}

The high vs low levels were categorized using the median value as the cut-off value

SPCS Severe postoperative complications, ASA American Society of Anesthesiologists, CRP C-reactive protein, TNF-a Tumor necrosis factor-a, AFR Albumin-tofibrinogen ratio, $O R$ Odds ratio, $\mathrm{Cl}$ Confidence interval

${ }^{*} P$ value $<0.05$ 
recommended as a prognostic factor in various models, e.g. acute ST-segment elevation myocardial infarction (STEMI) [39], operable NSCLC [15], and operable soft tissue sarcoma [40]. Preoperative low serum Alb is reported to be an indicator for postoperative complications and mortality in patients undergoing transcatheter aortic valve replacement [41]. Furthermore, early decrease in Alb is a significant predictor for SPCs in colorectal cancer patients undergoing curative laparoscopic surgery [42]. Previous studies have also indicated Fib as an early marker of postoperative complications after laparoscopic sleeve gastrectomy in morbidly obese patients [43] or total joint arthroplasty [44]. This present study was the first to indicate preoperative AFR as an independent risk factor for SPCs in GC patients after radical laparoscopic gastrectomy. The close association between inflammation and SPCs might be a possible mechanism.

This study had some certain limitations. First, this is a single-center study with the retrospective nature, so selection bias is inevitable. An independent prospective cohort is required to validate a definitive conclusion regarding clinical application of AFR and its optimal cutoff for SPCs prediction in surgical GC patients. Second, this study only takes preoperative Alb and Fib into consideration, whether postoperative levels have the predictive value remains unclear. Furthermore, the involved mechanisms for this study remain uncertain. A multicenter study with larger sample size was required to validate the prognostic role of AFR in GC patients. Furthermore, whether the interventions of AFR (e.g. improve the nutritional status, hypoproteinemia, coagulation function) could improve the outcomes in GC patients and decrease SPCs remains unclear.

\section{Conclusions}

To the best of our knowledge, this study firstly highlighted that preoperative AFR and age were two independent risk factors for SPCs in elderly surgical GC patients. Of course, our results do not support the delaying of elective surgery according to the preoperative AFR values. Instead, the situations with potential development of SPCs should be considered and intensively cared.

\section{Abbreviations}

AFR: Albumin-to-fibrinogen ratio; ASA: American Society of Anesthesiologists; BMI: Body mass index; Cl: Confidence interval; CRP: C-reactive protein: GC: Gastric cancer; Hb: Hemoglobin; Hct: Hematocrit; IL-6: Interleukin-6; NSCLC: Non-small cell lung cancer; OR: Odds ratio; Plt: Platelet; SPCs: Severe postoperative complications; STEMI: ST-segment elevation myocardial infarction; TNF-a: Tumor necrosis factor-a; WBC: White blood cell

\section{Acknowledgements \\ None.}

\section{Authors' contributions}

HS was involved in the caring for the patients included in the study, study design and methodology, interpretation and analysis of study results, and the writing of the manuscript. QZ was involved in identification and selection of patients, data acquisition, construction of the database, interpretation and analysis of study results, and administrative support. JS was involved in construction of the database, data acquisition, and administrative support. LG was involved in all statistical analysis, and writing of the manuscript. JC and XY edited the manuscript and supervised the study. All authors read and approved the final manuscript.

Funding

Medical Scientific Research Foundation of Zhejiang Province, China (No. 2019KY172) in the design of the study.

\section{Availability of data and materials}

Please contact the author Huachun Shen (shenhuachun_nb@sina.com) upon reasonable requests.

Ethics approval and consent to participate

This study was approved by the Medical Institutional Ethics Committee of Zhejiang province. The patients enrolled all presented written informed consent.

Consent for publication

Not Applicable.

\section{Competing interests}

The authors declare that they have no competing interests.

\section{Author details}

'Department of Anesthesiology, Jiangxi Maternal and Child Health Hospital, Nanchang, China. ${ }^{2}$ Department of Anesthesiology, HwaMei Hospital, University of Chinese Academy of Sciences, No.41 Xibei Road, Haishu District, Ningbo, Zhejiang Province, China.

Received: 18 May 2019 Accepted: 9 September 2019

Published online: 18 September 2019

References

1. Torre LA, Bray F, Siegel RL, Ferlay J, Lortet-Tieulent J, Jemal A. Global cancer statistics, 2012. CA Cancer J Clin. 2015;65(2):87-108.

2. Van Cutsem E, Sagaert X, Topal B, Haustermans K, Prenen H. Gastric cancer. Lancet. 2016;388(10060):2654-64.

3. Sasahara M, Kanda M, Ito S, Mochizuki $Y$, Teramoto H, Ishigure $K$, et al. The preoperative prognostic nutritional index predicts short-term and long-term outcomes of patients with stage II/III gastric cancer: analysis of a multiinstitution dataset. Dig Surg. 2019:1-10. https://doi.org/10.1159/000497454.

4. Yau GL, Silva PS, Arrigg PG, Sun JK. Postoperative complications of pars Plana vitrectomy for diabetic retinal disease. Semin Ophthalmol. 2018;33(1): $126-33$

5. Imamura H, Kurokawa Y, Kawada J, Tsujinaka T, Takiguchi S, Fujiwara Y, et al. Influence of bursectomy on operative morbidity and mortality after radical gastrectomy for gastric cancer: results of a randomized controlled trial. World J Surg. 2011;35(3):625-30.

6. Papamichael D, Audisio RA, Glimelius B, de Gramont A, Glynne-Jones R, Haller $\mathrm{D}$, et al. Treatment of colorectal cancer in older patients: international society of geriatric oncology (SIOG) consensus recommendations 2013. Ann Oncol. 2015;26(3):463-76.

7. Fleck A, Raines G, Hawker F, Trotter J, Wallace PI, Ledingham IM, et al. Increased vascular permeability: a major cause of hypoalbuminaemia in disease and injury. Lancet. 1985;1 (8432):781-4.

8. Toiyama Y, Yasuda H, Ohi M, Yoshiyama S, Araki T, Tanaka K, et al. Clinical impact of preoperative albumin to globulin ratio in gastric cancer patients with curative intent. Am J Surg. 2017;213(1):120-6.

9. Yamashita K, Ushiku H, Katada N, Hosoda K, Moriya H, Mieno H, et al. Reduced preoperative serum albumin and absence of peritoneal dissemination may be predictive factors for long-term survival with advanced gastric cancer with positive cytology test. Eur J Surg Oncol. 2015; 41(10):1324-32.

10. Liu ZJ, Ge XL, Ai SC, Wang HK, Sun F, Chen L, et al. Postoperative decrease of serum albumin predicts short-term complications in patients undergoing gastric cancer resection. World J Gastroenterol. 2017;23(27):4978-85. 
11. Kang SC, Kim HI, Kim MG. Low serum albumin level, male sex, and total gastrectomy are risk factors of severe postoperative complications in elderly gastric cancer patients. J Gastric Cancer. 2016;16(1):43-50.

12. Kijima T, Arigami T, Uchikado Y, Uenosono Y, Kita Y, Owaki T, et al. Combined fibrinogen and neutrophil-lymphocyte ratio as a prognostic marker of advanced esophageal squamous cell carcinoma. Cancer Sci. 2017; 108(2):193-9.

13. Kanda M, Tanaka C, Kobayashi D, Mizuno A, Tanaka Y, Takami H, et al. Proposal of the coagulation score as a predictor for short-term and longterm outcomes of patients with resectable gastric cancer. Ann Surg Oncol. 2017;24(2):502-9.

14. Guan $X$, Gong $M$, Wang $X$, Zhu J, Liu Y, Sun L, et al. Low preoperative fibrinogen level is risk factor for neurological complications in acute aortic dissection. Medicine (Baltimore). 2018;97(21):e10830.

15. Ying J, Zhou D, Gu T, Huang J, Liu H. Pretreatment albumin/fibrinogen ratio as a promising predictor for the survival of advanced non small-cell lung cancer patients undergoing first-line platinum-based chemotherapy. BMC Cancer. 2019;19(1):288.

16. Li SQ, Jiang YH, Lin J, Zhang J, Sun F, Gao QF, et al. Albumin-to-fibrinogen ratio as a promising biomarker to predict clinical outcome of non-small cell lung cancer individuals. Cancer Med. 2018;7(4):1221-31.

17. Japanese Gastric Cancer A. Japanese gastric cancer treatment guidelines 2010 (ver. 3). Gastric Cancer. 2011;14(2):113-23.

18. In H, Solsky I, Palis B, Langdon-Embry M, Ajani J, Sano T. Validation of the 8th edition of the AJCC TNM staging system for gastric cancer using the national cancer database. Ann Surg Oncol. 2017;24(12):3683-91.

19. Cao X, Zhao G, Yu T, An Q, Yang H, Xiao G. Preoperative prognostic nutritional index correlates with severe complications and poor survival in patients with colorectal cancer undergoing curative laparoscopic surgery: a retrospective study in a single Chinese institution. Nutr Cancer. 2017;69(3):454-63.

20. Clavien PA, Barkun J, de Oliveira ML, Vauthey JN, Dindo D, Schulick RD, et al. The Clavien-Dindo classification of surgical complications: five-year experience. Ann Surg. 2009;250(2):187-96.

21. Zhang WT, Lin J, Chen WS, Huang YS, Wu RS, Chen XD, et al. Sarcopenic obesity is associated with severe postoperative complications in gastric cancer patients undergoing gastrectomy: a prospective study. J Gastrointest Surg. 2018;22(11):1861-9.

22. Fukuda $Y$, Yamamoto $K$, Hirao M, Nishikawa K, Nagatsuma $Y$, Nakayama T, et al. Sarcopenia is associated with severe postoperative complications in elderly gastric cancer patients undergoing gastrectomy. Gastric Cancer. 2016;19(3):986-93.

23. Lo SL, Yen YH, Lee PJ, Liu CC, Pu CM. Factors influencing postoperative complications in reconstructive microsurgery for head and neck cancer. J Oral Maxillofac Surg. 2017;75(4):867-73.

24. Saji H, Ueno T, Nakamura H, Okumura N, Tsuchida M, Sonobe M, et al. A proposal for a comprehensive risk scoring system for predicting postoperative complications in octogenarian patients with medically operable lung cancer: JACS1303. Eur J Cardiothorac Surg. 2018;53(4):835-41.

25. Pedrazzani C, Moro M, Mantovani G, Lazzarini E, Conci S, Ruzzenente A, et al. C-reactive protein as early predictor of complications after minimally invasive colorectal resection. J Surg Res. 2017;210:261-8.

26. Miki Y, Toyokawa T, Kubo N, Tamura T, Sakurai K, Tanaka H, et al. C-reactive protein indicates early stage of postoperative infectious complications in patients following minimally invasive esophagectomy. World J Surg. 2017; 41(3):796-803.

27. Vasunilashorn SM, Dillon ST, Inouye SK, Ngo LH, Fong TG, Jones RN, et al. High C-reactive protein predicts delirium incidence, duration, and feature severity after major noncardiac surgery. J Am Geriatr Soc. 2017;65(8):e109-e16.

28. Mujagic E, Marti WR, Coslovsky M, Zeindler J, Staubli S, Marti R, et al. The role of preoperative blood parameters to predict the risk of surgical site infection. Am J Surg. 2018;215(4):651-7.

29. Chen YT, Ma FH, Wang CF, Zhao DB, Zhang YW, Tian YT. Elderly patients had more severe postoperative complications after pancreatic resection: a retrospective analysis of 727 patients. World J Gastroenterol. 2018;24(7):844-51.

30. Yu J, Hu J, Huang $C$, Ying $M$, Peng $X$, Wei $H$, et al. The impact of age and comorbidity on postoperative complications in patients with advanced gastric cancer after laparoscopic D2 gastrectomy: results from the Chinese laparoscropic gastrointestinal surgery study (CLASS) group. Eur J Surg Oncol. 2013;39(10):1144-9.

31. Kass KS, Velez-Cubian FO, Zhang WW, Toosi K, Tanvetyanon T, Rodriguez KL, et al. Effect of advanced age on peri-operative outcomes after robotic- assisted pulmonary lobectomy: retrospective analysis of 287 consecutive cases. J Geriatr Oncol. 2017;8(2):102-7.

32. Gretschel S, Estevez-Schwarz L, Hunerbein M, Schneider U, Schlag PM. Gastric cancer surgery in elderly patients. World J Surg. 2006;30(8):1468-74.

33. Ritz P. Physiology of aging with respect to gastrointestinal, circulatory and immune system changes and their significance for energy and protein metabolism. Eur J Clin Nutr. 2000;54(Suppl 3):S21-5.

34. Polanczyk CA, Marcantonio E, Goldman L, Rohde LE, Orav J, Mangione CM, et al. Impact of age on perioperative complications and length of stay in patients undergoing noncardiac surgery. Ann Intern Med. 2001;134(8):637-43.

35. Artigas A, Wernerman J, Arroyo V, Vincent JL, Levy M. Role of albumin in diseases associated with severe systemic inflammation: pathophysiologic and clinical evidence in sepsis and in decompensated cirrhosis. J Crit Care. 2016;33:62-70.

36. Gupta D, Lis CG. Pretreatment serum albumin as a predictor of cancer survival: a systematic review of the epidemiological literature. Nutr J. 2010;9:69.

37. Jensen T, Kierulf P, Sandset PM, Klingenberg O, Joo GB, Godal HC, et al. Fibrinogen and fibrin induce synthesis of proinflammatory cytokines from isolated peripheral blood mononuclear cells. Thromb Haemost. 2007;97(5):822-9.

38. Sheng L, Luo M, Sun X, Lin N, Mao W, Su D. Serum fibrinogen is an independent prognostic factor in operable nonsmall cell lung cancer. Int J Cancer. 2013:133(11):2720-5.

39. Zhao Y, Yang J, Ji Y, Wang S, Wang T, Wang F, et al. Usefulness of fibrinogen-to-albumin ratio to predict no-reflow and short-term prognosis in patients with ST-segment elevation myocardial infarction undergoing primary percutaneous coronary intervention. Heart Vessels. 2019;34(10): 1600-7.

40. Liang Y, Wang W, Que Y, Guan Y, Xiao W, Fang C, et al. Prognostic value of the fibrinogen/albumin ratio (FAR) in patients with operable soft tissue sarcoma. BMC Cancer. 2018;18(1):942.

41. Gassa A, Borghardt JH, Maier J, Kuhr K, Michel M, Ney S, et al. Effect of preoperative low serum albumin on postoperative complications and early mortality in patients undergoing transcatheter aortic valve replacement. J Thorac Dis. 2018;10(12):6763-70.

42. Wang $Y$, Wang $H$, Jiang J, Cao X, Liu Q. Early decrease in postoperative serum albumin predicts severe complications in patients with colorectal cancer after curative laparoscopic surgery. World J Surg Oncol. 2018;16(1):192.

43. Ruiz-Tovar J, Munoz JL, Gonzalez J, Garcia A, Ferrigni C, Jimenez M, et al. Creactive protein, fibrinogen, and procalcitonin levels as early markers of staple line leak after laparoscopic sleeve gastrectomy in morbidly obese patients within an enhanced recovery after surgery (ERAS) program. Surg Endosc. 2017;31(12):5283-8.

44. Oelsner WK, Engstrom SM, Benvenuti MA, An TJ, Jacobson RA, Polkowski GG, et al. Characterizing the acute phase response in healthy patients following total joint arthroplasty: predictable and consistent. J Arthroplast. 2017;32(1):309-14.

\section{Publisher's Note}

Springer Nature remains neutral with regard to jurisdictional claims in published maps and institutional affiliations.

Ready to submit your research? Choose BMC and benefit from:

- fast, convenient online submission

- thorough peer review by experienced researchers in your field

- rapid publication on acceptance

- support for research data, including large and complex data types

- gold Open Access which fosters wider collaboration and increased citations

- maximum visibility for your research: over $100 \mathrm{M}$ website views per year

At $\mathrm{BMC}$, research is always in progress.

Learn more biomedcentral.com/submission 